\title{
5 ,The Missing Link“ zwischen Individuum und Gesellschaft - die Psychoanalyse als politische Wissenschaft
}

\subsection{Die exemplarische Darstellung sozioökonomischer Verhältnisse in der Psychoanalyse der Zwischenkriegszeit von Freud bis Reich}

In diesem Kapitel soll die Frage beantwortet werden, wie die sozioökonomischen Verhältnisse Eingang in die Theorienbildung der Psychoanalyse der Zwischenkriegszeit gefunden haben. Dabei wird - neben Freud - auf Bernfeld, Fenichel, Fromm und Reich genauer eingegangen, die mit ihren Konzeptionen mein psychoanalytisches Verständnis für die Finanz- und Wirtschaftskrise beeinflusst haben. Von ihnen wurde die Psychoanalyse als Instrument zur Analyse und zum Verständnis gesellschaftlicher und kultureller Phänomene angewandt, so wie es auch Anliegen dieser Arbeit ist. In der Darstellung der sozioökonomischen Matrix der Finanzkrise 2008 wird auch auf diese Ausführungen zurückgegriffen.

\subsubsection{Sigmund Freud}

Nach der russischen Revolution im Jahr 1917 fand der Marxismus noch weitere Verbreitung und wurde damit unweigerlich zu einer gesellschaftlichen Kraft, die zu einer theoretischen Auseinandersetzung herausforderte. Daneben verschiebt sich die Blickrichtung vom Individuum auf Massenphänomene und Institutionen, wobei Letztere maßgeblich für die Produktion von Unbewusstheit verantwortlich sind. „Die Ende der zwanziger Jahre begonnene Weltanschauungsdebatte stand im Kontext zu den politischen und ökonomischen Kämpfen $[\ldots]$ “. (Friedrich, 1987, S. 208).

In der 35. Vorlesung „Über eine Weltanschauung“, die 1933 als Teil der „Neuen Folge der Vorlesungen zur Einführung in die Psychoanalyse“ veröffentlicht wurde, setzt sich Freud kritisch mit verschiedenen Weltanschauungen auseinander; darunter mit der Religion, die von den „drei Mächten, die der Wissenschaft Grund und Boden bestreiten können [...] allein der ernsthafte Feind" ist (S. Freud, 1933a, S. 588). Im Gegensatz zu Kunst und Philosophie, so Freud, hält die Religion nicht nur ein Welterklärungsmodell bereit, sondern vermag durch den immanenten Trost und die Fürsorge, die an die Einhaltung von Regeln 
gebunden und den familiären Beziehungen zwischen Eltern und Kind nachgebildet ist, etwas, das die Wissenschaft nicht leisten kann.

Im Anschluss daran wendet sich Freud dem Marxismus als einer Weltanschauung zu, gesteht aber gleichzeitig ein, dass ihm ,die richtige Kompetenz zu deren Beurteilung abgeht" (S. Freud, 1933a, S. 601). Augenscheinlich ist, dass die Auseinandersetzung mit dem Marxismus nicht nur aus theoretischer Sicht vonnöten war; sie hatte auch mit Reich zu tun, worauf später noch eingegangen wird. Wesentlich sind die Lösungsversprechen und Zukunftsvorstellungen, die gerade unter den verarmten und sozial deprivierten Menschen Hoffnung erzeugen und denen Freud kritisch begegnet:

„Er [der Marxismus] hofft, im Laufe weniger Generationen die menschliche Natur so zu verändern, daß sich ein fast reibungsloses Zusammenleben der Menschen in der neuen Gesellschaftsordnung ergibt und daß sie die Aufgaben der Arbeit zwangsfrei auf sich nehmen. Unterdes verlegte er die in der Gesellschaft unerläßlichen Triebeinschränkungen an andere Stellen und lenkt die aggressiven Neigungen, die jede menschliche Gemeinschaft bedrohen, nach außen ab, stürzt sich auf die Feindseligkeit der Armen gegen die Reichen, der bisher Ohnmächtigen gegen die früheren Machthaber " (S. Freud, 1933a, S. 606).

Die Nähe zur Religion ergibt sich durch Freud auch durch das Verbot einer Kritik: „Die Werke von Marx haben als Quelle einer Offenbarung die Stelle der Bibel und des Korans eingenommen, obwohl sie nicht freier von Widersprüchen und Unklarheiten sein sollen als diese älteren heiligen Bücher" (S. Freud, 1933a, S. 606). Freud kritisiert die Notwendigkeit oder Naturgesetzlichkeit der Entwicklung der Gesellschaft im Marx'schen Sinne, die ihm „eher wie ein Niederschlag jener dunkeln Hegelschen Philosophie“ (S. Freud, 1933a, S. 603) erscheint. Stattdessen führt er die sozialen Unterschiede auf ursprüngliche Rassen- und Stammesunterschiede zurück, auf konstitutionelle Faktoren und auf die zunehmende Naturbeherrschung, die sich in technischen Errungenschaften widerspiegelt (vgl. S. Freud, 1933a, S. 604).

Hier findet sich eine Passage zur Wirtschaftskrise, die ein wenig kryptisch anmutet oder, wie Freud selbst feststellt, nicht plausibel klingt.

„Ja, vielleicht zahlen wir mit der gegenwärtigen, an den Weltkrieg anschließenden Wirtschaftskrise auch nur den Preis für den letzten großartigen Sieg über die Natur, die Eroberung des Luftraums. Das klingt nicht sehr einleuchtend, aber wenigstens die ersten Glieder des Zusammenhangs sind klar zu erkennen. Die Politik Englands fußte auf der Sicherheit, die ihm das seine Küsten umspülende Meer verbürgte. Im Moment, da Bleriot den Kanal im Aeroplan überflogen hatte, war diese schützende Isolierung durchbrochen, und in jener Nacht als in Friedenszeiten und zu Übungszwecken ein deutscher Zeppelin über London kreiste, war wohl der Krieg gegen Deutschland beschlossene Sache“ (S. Freud, 1933a, S. 604). 
Technische Errungenschaften verändern die sozialen, nationalen und damit die ökonomischen Verhältnisse in einem größeren Maße, als angenommen wird.

In einem Brief an Arnold Zweig vom 26. 11. 1930 definiert sich Freud als „Liberaler vom alten Schlag“, dem bei ,aller Unzufriedenheit mit den gegenwärtigen Wirtschaftsordnungen [...] doch jede Hoffnung, daß der von den Sowjets eingeschlagene Weg zur Besserung führen wird“ (E. L. Freud, 1980, S. 33), fehle.

Für Freud - und damit löst er die Psychoanalyse von der Weltanschauung ist Erstere „ein Stück Wissenschaft [...]. [...] sie schaut nicht alles an, sie ist zu unvollendet, erhebt keinen Anspruch auf Geschlossenheit und Systembildung" (S. Freud, 1933a, S. 608). Und an anderer Stelle präzisiert er den Zusammenhang im Vorgehen:

„Der Fortschritt in der wissenschaftlichen Arbeit vollzieht sich ganz ähnlich wie in einer Analyse. Man bringt Erwartungen in die Arbeit mit ein, aber man muß sie zurückdrängen. Man erfährt durch die Beobachtung bald hier, bald dort etwas Neues, die Stücke passen zunächst nicht zusammen. Man stellt Vermutungen auf, macht Hilfskonstruktionen, die man zurücknimmt, wenn sie sich nicht bestätigen, man braucht viel Geduld, Bereitschaft für alle Möglichkeiten, verzichtet auf frühe Überzeugungen, um nicht unter deren Zwang neue, unerwartete Momente zu übersehen, und am Ende lohnt sich der ganze Aufwand, die zerstreuten Funde fügen sich zusammen, man gewinnt den Einblick in ein ganzes Stück des seelischen Geschehens [...] “ (S. Freud, 1933a, S. 600 f.).

Freud wollte die Psychoanalyse aus der politischen Debatte heraushalten ${ }^{62}$, indem er ihren szientistischen wissenschaftlichen Charakter betonte, wogegen Bernfeld, aber noch viel mehr Reich einen anderen Standpunkt vertraten, wie im Folgenden ausgeführt wird.

\subsubsection{Siegfried Bernfeld}

„[...] die Ermordung des Urvaters Wallstreet und die Besitzergreifung der Urmutter Boden und Kapital ist durch Schuldgefühl und Angst [...] gesichert" (Bernfeld, 1973, S. 109).

Bernfeld (1928) widmet sich in seinem Aufsatz „Ist die Psychoanalyse eine Weltanschauung?" explizit diesem Thema.

Bernfeld gehört mit seiner Konzeption des „,sozialen Ortes“ zu den interessantesten Theoretikern rund um Freud. Er schärft den Blick auf die gesellschaftlichen Faktoren der Krankheitsgenese und Theoriebildung und weist stellvertre-

62 Vergleiche dazu auch Jacoby (1985, S. 103, 128 f.). 
tend für Freud den Vorwurf des fehlenden Interesses der Psychoanalyse für das Soziale zurück, wenn er schreibt:

„Ist doch alles seelische Geschehen für Freud das Schicksal, das die Triebe in einer bestimmten Realität finden; die Triebe selbst mitsamt ihren Eigenschaften und Zielen sind der Niederschlag historischen Geschehens, und wo nicht die Realität, sondern innere Faktoren für das Triebschicksal entscheidend sind, da ist das Über-Ich wirksam, das selbst nur die Verinnerlichung eines bedeutsamen Stücks der Realität ist" (Bernfeld, 1974a, S. 210).

Damit reagiert er auf Angriffe, die das Bürgertum und dessen spezifische Probleme betreffen, aber auch auf die Zeitumstände, die eine Stellungnahme fordern.

Bernfeld präzisiert die sozialen Einflussfaktoren, wenn er am Beispiel einzelner Patientenschicksale nachweist, wie sehr ein bestimmtes Milieu ein spezifisches Leiden erzeugt, dessen subjektive Bewertung beim Betroffenen und in der Einschätzung der Umgebung je nach ,sozialem Ort“ differieren kann.

„Innerhalb desselben Zeitalters erfahren die Triebschicksale unbeschadet der Gültigkeit aller Freudschen Mechanismen und Dynamismen je nach dem Milieu, in dem sie ablaufen, ihre eigenartige Prägung ${ }^{63}$ [...] Man kann die Fragestellung nach dem historischen Aspekt und nach der Milieuprägung eines seelischen Vorgangs als den Gesichtspunkt des , sozialen Orts ${ }^{64}$ zusammenfassen und hervorheben " (Bernfeld, 1974a, S. 210).

Als Beispiel werden die Bewertung der Homosexualität und das damit verbundene Leiden in verschiedenen Gesellschaften, Kulturen und Zeiten oder das Leiden in unglücklichen Ehen angeführt, wobei Letzteres differieren kann, wenn es nicht unausweichlich erscheint.

„Als neurotisches Leiden wäre dann solches zu bezeichnen, das vom Über-Ich ausgeht, ohne daß es sozial gefordert wäre [...] Soweit die Bedingungen des sozialen Ortes der Beteiligten unveränderbar, notwendig erscheinen, ist auch das Leid so unvermeidlich wie der Schmerz. Wem aber die sozialen Bedingungen änderbar scheinen, der wehrt sich gegen das , sinnlose "überflüssige Leid" (Bernfeld, 1974a, S. $212 f$.).

Dass damit sowohl Weltanschauungen als auch Therapierichtungen ein Problem haben, kommentiert Bernfeld maliziös:

63 Hervorhebung durch Bernfeld.

64 Hervorhebung durch Bernfeld. 
„Wer die gegebene Gesellschaftsordnung, die so viel Leid stiftet, absolut akzeptiert, daher die Änderbarkeit des Leids nicht lehren kann und auch das neurotische Leiden wegen unzulänglicher Methode nicht zu verringern vermag, muß versuchen, den Patienten mit Leid und Leiden abzufinden, indem er ihm einen Sinn seines Lebens, eine Aufgabe für sein Leben zu vermitteln trachtet" (Bernfeld, 1974a, S. 213 f.).

Ein Wechsel des sozialen Orts kann äußerlich gesehen mit Aufstieg oder Abstieg gleichgesetzt werden, psychodynamisch aber auch den Verdrängungs- und Sublimierungsaufwand verringern, wie Bernfeld am Beispiel eines jungen Aristokraten zeigt, der aus der Sicht seiner Familie besorgniserregend verwahrloste, sich als Kommunist letztlich aber von den Anforderungen der sozialen Stellung befreite und über die revolutionären Vorstellungen noch dazu seine aggressiven Tendenzen gegenüber den Elternfiguren in der Familie befriedigen konnte. Ein weiteres Beispiel sind Jugendliche, die aus einem proletarischen Milieu kommen, ihr Heim oder die Arbeitsstelle verlassen und auf der Straße leben. Im Gegensatz zum bürgerlichen Kind verlieren diese Jugendlichen, so Bernfeld, „keine Nahrungs- und Kleidungsprämien, keine geliebten Phantasierplätze, kein Eigentum, kein Spielzeug, Bücher, keine geliebten Erzieher“ (Bernfeld, 1974a, S. 223). Der proletarische Jugendliche habe auch nichts zu verlieren. „So setzt der soziale Ort für das bürgerliche und proletarische Kind je eine andere Chance zur Entwicklung.“65 (Bernfeld, 1974a, S. 223)

In seinem Aufsatz „Die Tantalussituation“ (1931) setzt sich Bernfeld (1974b) mit dem „kriminellen Über-Ich“ auseinander, das von Alexander und Staub bei bestimmten Taten „von normalen Kriminellen“ ins Treffen geführt wird, indem er die Tantalussituation des spezifischen sozialen Ortes ins Treffen führt, an dem Entbehrungen, Armut und ökonomische Enge, wie schon weiter oben dargestellt, auf der Tagesordnung stehen, während das Großstadtleben alle möglichen Befriedigungsmöglichkeiten bietet. Auch diese Jugendlichen haben nichts zu verlieren.

„Sie werden weniger abgeschreckt, weil in ihrer Situation rechtschaffen zu sein real nicht lohnt; es bringt keine entsprechenden sozialen Kompensationen. Die Triebeinschränkungen an diesem Ort werden in wachsendem Maße nur mehr durch das Über-Ich, also durch die Gewissensangst und immer weniger auch durch die Realangst gesichert" (Bernfeld, 1974b, S. 343).

Das Symptom kann also auch durch klassenspezifische Bedingungen hervor gebracht werden. So meint Bernfeld, dass die nach Freud in der Ätiologie der psychischen Erkrankungen zu untersuchenden konstitutionellen und biografi-

65 Hervorhebung durch Bernfeld. 
schen Faktoren um den Faktor des sozialen Orts ergänzt werden müssten (vgl. Bernfeld, 1974b, S. 345).

Eine geringe Realangst sei auch politischen Bewegungen zuzuschreiben, die mit dem herrschenden Gesellschaftssystem nicht mehr zufrieden sind. In diesem Punkt beweist Bernfeld einen differenzierten Weitblick, wenn er schreibt:

„Bis vor kurzem waren diese Gesellschaftskräfte durch den Sozialismus (die Arbeiterbewegung) repräsentiert. Wir sehen in den letzten Jahren die Anerkennung der radikalen Änderungsbedürftigkeit der Gesellschaft weit über diesen Kreis hinauswachsen, zum Beispiel als Faschismus " (Bernfeld, 1974b, S. 343 f.).

Auch die geistigen Produkte einer Kultur, die Wissenschaften, werden je nach sozialem Ort unterschiedlich rezipiert und ausgeformt. Bernfeld unterzieht die Psychologie und die Psychotherapie einer Kritik, die sich auf deren „Konjunktur" und „Lebensnähe“ bezieht, und wendet sich gegen ihre politische und ökonomische Vereinnahmung. Im folgenden Textausschnitt richtet sich die Polemik gegen die Individualpsychologie, die sozioökonomische Gegebenheiten dem Individuum anlastet. Damit macht sich die Psychoanalyse nicht beliebt.

„Der Psychologe hört sich gerufen. Der Konzern-Aufsichtsrat ruft ihn zur Auslese der wirtschaftsbegabten Angestellten, die so abgebauten Angestellten rufen ihn zur Behebung ihrer Minderwertigkeit, zum Aufbau des Gemeinschaftsgefühls im Du" (Bernfeld, 1974c, S. 206).

Bernfeld ist in seiner Analyse der Wissensproduktion in Abhängigkeit von realen Gegebenheiten und Bedürfnissen hoch aktuell.

„Es sind sehr klare Fragestellungen, die die Wirtschaft an den Psychologen stellt, sie verlangt von ihm Rationalisierung des Betriebes, der Menschenbeeinflussung, -Erziehung, -Auslese; das riesige Gebiet der angewandten Psychologie verdankt seinen imponierenden rapiden Ausbau diesem kommerziellen Bedürfnis. Es macht diese Zweige der Psychologie zu naturwissenschaftlichen, es zwingt sie zur Rationalität der Methoden, zur Klärung der Kriterien, zur Exaktheit der Ergebnisse [...] Damit aber binden sich die wissenschaftlichen Interessen dieser Zweige der Psychologie [...] an die Prosperität der Wirtschaft" (Bernfeld, 1974c, S. 205 f.).

\subsubsection{Wilhelm Reich}

In diesem Kapitel soll zunächst Reichs Analyse der gesellschaftspolitischen Struktur der Zwischenkriegszeit in den Fokus gestellt werden, da sie in dieser Form ein einzigartiges Zeitdokument darstellt. In einem eigenen Abschnitt wird am Beispiel Wilhelm Reich die Verbindung von Psychoanalyse und Gesellschafts- und Institutionenpolitik in jener Zeit näher untersucht werden. Sie spie- 
gelt die Brisanz dieses Verhältnisses wider und erhellt den gespaltenen Umgang miteinander.

\subsubsection{1 „Die Massenpsychologie des Faschismus“ als Spiegel der wirtschaftspolitischen Situation in den 1930er-Jahren}

Reich war einer der wenigen Psychoanalytiker der ersten Generation, der in seinen Analysen die wirtschaftliche und politische Situation jener Zeit in $\mathrm{Zu}$ sammenhang mit der ideologischen Struktur gesellschaftlicher Schichten und der charakterlichen Struktur von Menschen brachte und auf diese Weise gegenwärtig - im doppeldeutigen Sinn - die Bedingungen analysierte, die dem Faschismus den Boden bereiteten. ${ }^{66}$ Dokumentiert ist etwa ein Vortrag Reichs am Berliner Institut mit dem Titel „Massenpsychologische Probleme innerhalb der Wirtschaftskrise" vom 28. 6. 1932 (vgl. A. Freud, 1932, S. 559 f.).

Eine umfassende Darstellung der gegenseitigen Befruchtung, Bekämpfung und Abgrenzung von Marxismus und Psychoanalyse findet man bei Dahmer (2013a).

In der Zeit zwischen 1928 und 1933, der Zeit der Weltwirtschaftskrise, ergab sich nach Reich folgendes Bild:

„, [...] das Kapital war in wenigen Händen konzentriert, die Entwicklung der Nationalwirtschaft zur Weltwirtschaft stand in schärfstem Widerspruch zum Zollsystem der nationalen Staaten, die kapitalistische Wirtschaft erreichte die Produktionskapazität kaum zur Hälfte und hatte ihre Anarchie restlos enthüllt. Die Mehrheit der Bevölkerung der hochindustriellen Länder war verelendet, etwa 50000000 Menschen waren in Europa arbeitslos, hunderte Millionen Schaffender fristeten ein Hungerdasein " (Reich, 1986, S. 32).

Folgt man der Theorie von Marx, wären alle ökonomischen Voraussetzungen erfüllt gewesen, die zur Revolution hätten führen sollen. Und dennoch wurde der Nationalsozialismus und nicht der Marxismus zu einer Massenbewegung. Nach Reich hatten die Marxisten die Rolle des Kleinbürgertums, das durch die Wirtschaftskrise vom sozialen Abstieg bedroht war, als entscheidenden Faktor vernachlässigt; die ökonomischen Analysen, die für das 19. Jahrhundert zutrafen, waren in dieser Form nicht mehr realitätsgerecht. Die Marxisten hatten auch keine Erklärung dafür,

66 Im Vorwort zur dritten korrigierten Auflage aus dem Jahr 1942 schreibt Reich: „Die Massenpsychologie des Faschismus entstand in den deutschen Krisenjahren 1930 1933 [...] Die Faschisten verboten das Buch 1935 zusammen mit der gesamten Literatur der politischen Psychologie“ (Reich, 1986, S. 17). 
„, daß die wirtschaftliche Krise, die der Erwartung nach eine Linksentwicklung der Ideologie der Massen hätte mit sich bringen müssen, zu einer extremen Rechtsentwicklung in der Ideologie der proletarisierten Schichten der Bevölkerung geführt hatte. Es ergab sich eine Schere zwischen der Entwicklung in der ökonomischen Basis, die nach links drängte, und der Entwicklung der Ideologie breiter Schichten nach rechts" (Reich, 1986, S. 30 f.).

Wie es zu dieser Entwicklung kommen konnte, in der ökonomische Basis und ideologische Struktur so weit auseinanderklafften - diesen Widerspruch aufzulösen, geht Reich in diesem Buch nach. Nach Reich war es zwischen 1927 und 1933 ein Versäumnis der Theorie und Praxis des Marxismus, dass man zwar die ökonomischen Gegebenheiten, nicht aber die psychologische Dimension, „die charakterliche Struktur der Massen und die soziale Wirkung des Mystizismus“, berücksichtigt habe (Reich, 1986, S. 29).

In seiner Auseinandersetzung mit dem sogenannten „Vulgärmarxismus“667, den Mängeln einer rein soziologischen und denen einer psychoanalytischen Betrachtungsweise, arbeitet er sukzessive heraus, wie die gesellschaftliche Struktur die Charakterbildung des Individuums beeinflusst und Letztere wieder auf Erstere zurückwirkt.

„Diese Lücke füllt die charakteranalytische Psychologie aus, indem sie den Prozess im menschlichen Seelenleben aufdeckt, der von den Seinsbedingungen bestimmt ist. Derart erfasst sie den subjektiven Faktor, den der Marxist nicht begreift [...] Sie untersucht zwar nur den einzelnen Menschen. Wenn sie sich aber zur Erforschung der einer Schichte, Klasse, Berufsgruppe etc. gemeinsamen, typischen psychischen Prozesse spezialisiert und das individuell Unterschiedliche ausschaltet, wird sie zur Massenpsychologie“ (Reich, 1986, S. 37 f.).

In einer Zusammenführung von Psychoanalyse und Marxismus entsteht die „sexualökonomische Soziologie“ Reichs, in der ,triebhafte und sozialökonomische Prozesse" (Reich, 1986, S. 22) das menschliche Sein bestimmen.

67 „Der Vulgärmarxismus trennt schematisch das wirtschaftliche Sein vom allgemeinen gesellschaftlichen Sein überhaupt ab und behauptet, dass die ,Ideologie‘ und das ,Bewusstsein' der Menschen durch das wirtschaftliche Sein allein und unmittelbar bestimmt werden. So gelangt er zu einer mechanistischen Gegenüberstellung von Wirtschaft und Ideologie, von ,Basis' und ,Überbau'; er macht die Ideologie schematisch und einseitig abhängig von der Wirtschaft und übersieht die Abhängigkeit der Entwicklung der Wirtschaft von der der Ideologie. Aus diesem Grunde ist ihm das Problem der sogenannten ,Rückwirkung' der Ideologie verschlossen“" (Reich, 1986, S. 36). 
„Die Psychoanalyse ist die Mutter, die Soziologie der Vater der Sexualökonomie. Aber ein Kind ist mehr als die Summe der Eltern"“68 (Reich, 1986, S. 22). Reichs Kritik an der Psychoanalyse richtet sich gegen die Auffassung der Gesellschaft als Summe von Individuen, weiters gegen den Gegensatz von Kultur und Natur des Menschen und die Verankerung der Destruktion im Individuum, wie sie durch die Annahme des Todestriebs in der letzten Triebtheorie Freuds etabliert wurde. ${ }^{69}$ Stattdessen macht er seine individuelle und soziale Sexualökonomie $^{70}$ zum Analyseinstrument gesellschaftlicher individueller Beziehungen, wie man am Beispiel seiner Charakteranalyse nachvollziehen kann.

Reich beschreibt die „Schichten der Charakterstruktur“ als ,autonom funktionierende Ablagerungen der sozialen Entwicklung" und unterscheidet dabei drei Schichten; in einer oberflächlichen Schicht zeigt sich der Mensch fähig zur Kooperation (Reich, 1986, S. 11). Zwischen dieser und dem sogenannten „biologischen Kern“, in dem nach Reich „,der Mensch ein unter günstigen sozialen Umständen ehrliches, arbeitsames, kooperatives, liebendes oder, wenn begründet, rational hassendes Tier“ ist, liegt die „perverssadistische Charakterschichte“, die Reich in Übereinstimmung mit Freud als das Unbewusste oder Verdrängte bezeichnet (Reich, 1986, S. 11).

Das heißt - und das ist von Bedeutung! -, dass der Mensch im Kern gut ist; im Unterschied zu Freud formuliert Reich in diesem Punkt ein positives Menschenbild, wogegen Freud von einem Antagonismus des Sexual- und des Todestriebs ausging, die in einen unauflöslichen Widerspruch zu den Anforderungen der Kultur treten und das „Unbehagen an der Kultur“ auslösen. ${ }^{71}$ Die sozioöko-

68 Hervorhebung durch Reich.

69 An dieser Stelle soll darauf verwiesen werden, dass Reich trotz der Kritik und der Anfeindungen, die ihm vonseiten der Psychoanalyse entgegenschlugen, diese selbst nicht aufnahm. „Sie [die analytische Soziologie] steht seit langem Bestrebungen, die diese Konsequenzen ziehen, feindlich gegenüber, und ihre Vertreter erweisen sich im Kampfe gegen solche Bestrebungen keineswegs inkonsequent. Das ändert nichts daran, daß wir die großen Freudschen Entdeckungen gegen jeden Angriff, von welcher Seite immer er kommen mag, aufs schärfste zu verteidigen entschlossen sind“" (Reich, 1986, S. 47).

70 Sie stellt zum Beispiel die Frage: „Aus welchem soziologischen Grunde wird die Sexualität von der Gesellschaft unterdrückt und vom Individuum zur Verdrängung gebracht?“" (Reich, 1986, S. 48).

71 Die Ansprüche der Kultur schränken nach Freud (1930a) das Individuum in seinem Streben nach Glück und Lust, aber auch in seinen destruktiven Impulsen massiv ein, sodass es nicht verwunderlich erscheint, dass sich Unzufriedenheit oder Unbehagen breit machen und das Individuum in realer Zerstörung bzw. in Ideologien oder religiösen Systemen, die die Wirklichkeit verleugnen, nach Erlösung sucht. Diese löschen die Widersprüche der Realität, die nach ständigen Anpassungen verlangt, 
nomische Struktur einer Gesellschaft spiegelt sich nach Reich in der Charakterstruktur ihrer Individuen, damit wird auch ihre prinzipielle Veränderbarkeit angezeigt. Dabei handelt es sich nicht um eine monokausale Einflussnahme, sondern um eine wechselseitige Bedingtheit von Charakterstruktur und sozialer Struktur, die aufeinander einwirken.

„Nachdem soziale Umstände und Veränderungen die ursprünglichen biologischen Ansprüche des Menschen zur Charakterstruktur geformt haben, reproduziert die Charakterstruktur in Form der Ideologien die soziale Struktur der Gesellschaft " (Reich, 1986, S. 12).

In seiner Analyse des Faschismus führt Reich die charakterliche Schichtung der Individuen mit den gesellschaftlichen Strukturen zusammen, die sich wechselseitig verstärken. Für Reich ist der Faschismus kein politisches, auch kein nationales Phänomen. Der Faschismus, als eine Massenbewegung, repräsentiert die zweite Ebene der charakterlichen Schichtung und ist „die emotionale Grundhaltung des autoritär unterdrückten Menschen der maschinellen Zivilisation und ihrer mechanistisch-mystischen Lebensauffassung" (Reich, 1986, S. 13). Der Faschismus ist Ausdruck eines „religiösen Mystizismus“, der auf einer autoritären Gesellschaftsstruktur aufbaut, in der Unterdrückung und Leiden in eine sadistische Religion umgewandelt werden (vgl. Reich, 1986, S. 14 f.).

In seiner Analyse des Kleinbürgertums wird von Reich offengelegt, warum dieses besonders anfällig für die nationalsozialistische Ideologie war. Von Absturzängsten aufgrund der wirtschaftlichen Situation geplagt, konnte die über Jahrhunderte entwickelte Verzahnung der Familie als Produktions- und Reproduktionsstätte nicht mehr aufrechterhalten werden. Sowohl innerfamiliär als auch in Identifikation mit den Obrigkeiten herrschte ein patriarchales Verständnis von Unterwerfung unter väterliche Autoritäten. Der Nationalsozialismus bediente sich eines religiösen Mystizismus, indem er den Wunsch nach Schutz durch die elterliche Autorität oder durch einen schützenden Vatergott auf den „Führer“ übertrug und sich der gehemmten sexuellen Energie bediente, die in sadistischen Entladungen gegenüber Juden, politisch Andersdenkenden und Randgruppen

real oder phantasmatisch aus. So wagt Freud am Ende seiner Abhandlung einen skeptischen Blick in die Zukunft:

„Die Schicksalsfrage der Menschenart scheint mir zu sein, ob und in welchem Maße es ihrer Kulturentwicklung gelingen wird, der Störung des Zusammenlebens durch den menschlichen Aggressions- und Selbstvernichtungstrieb Herr zu werden [...] Und nun ist zu erwarten, daß die andere der beiden , himmlischen Mächte', der ewige Eros, eine Anstrengung machen wird, um sich im Kampf mit seinem ebenso unsterblichen Gegner zu behaupten. Aber wer kann den Erfolg und Ausgang voraussehen?" (S. Freud, 1930a, S. 270). 
ihren Ausdruck fand. Das über Jahrhunderte kultivierte autoritäre Familiensystem, das auf Gehorsam und sexuelle Kontrolle der Frauen und Kinder ausgelegt ist, bewirkt eine Spaltung des Frauenbildes zwischen Mutter und Hure und eine unterwürfige Haltung gegenüber staatlichen Autoritäten. Während wirtschaftliche Not und Armut bewusst erlebt werden, werden die sexuellen Regungen verdrängt und äußern sich in Form von Ersatzbefriedigungen oder Verschiebungen. So ist es auch verständlich, dass sich statt des erwarteten Aufbegehrens der Menschen die Unterordnung unter einen autoritären Führer vollzog. „Die Sexualhemmung verändert den wirtschaftlich unterdrückten Menschen strukturell derart, daß er gegen sein materielles Interesse handelt, fühlt und denkt" (Reich, 1986, S. 51).

Das religiöse Gefühl ist für Reich nichts anderes als gehemmte sexuelle Energie, die nach Entladung drängt. „Das tiefe Sehnen nach Auslösung und Erlösung, bewu $\beta t^{72}$ von den ,Sünden', unbewu $\beta t^{73}$ von der sexuellen Spannung, ist gleichzeitig abgewehrt“ (Reich, 1986, S. 144). Für Reich ist Mystik „unbewusste Orgasmussehnsucht“" (Reich, 1986, S. 158).

In der Rassentheorie der Nationalsozialisten kommt es zu einer Spaltung wie schon bei der Spaltung des Frauenbildes in Mutter und Hure - in das eines reinen, von Sexualität befreiten Gefühls, das den Ariern zugeschrieben wird, und das des schmutzigen Sexuellen, das auf die Juden projiziert wird. Und Reich präzisiert, was dies für die Strukturierung der Gesellschaft bedeutet:

„So wird auch die Demokratisierung eines politischen Systems, die durch Mischehen zustande kommt, als Erscheinung des Niedergangs der Rasse gedeutet. [...] Angehörige der unterdrückten Klasse werden mit Fremdrassigen gleichgestellt “"74 (Reich, 1986, S. 100).

Hier wird deutlich, warum Reich in einer Zeit, als man die Psychoanalyse von der Politik trennen wollte, so gefährlich wurde.

„Kommunisten und Analytiker waren sich darin einig, dass Reich ihre eigene Lehre geschädigt habe, indem er sie mit der jeweils anderen verband [...] "(Peglau, 2013, S. 507).

Nach Andreas Peglau ist Reich „neben Erich Fromm der einzige Analytiker geblieben, der sich tiefgründiger um ein geschlossenes psychoanalytisches Verständnis des Faschismus bemühte“ (Peglau, 2013, S. 506).

72 Hervorhebung durch Reich.

73 Hervorhebung durch Reich.

74 Hervorhebung durch Reich. 


\subsubsection{Die Verbindung von Psychoanalyse und Politik am Beispiel Wilhelm Reichs}

„Ein faschistisches Regime, das zugelassen hätte, dass seine destruktiven, krankmachenden Aspekte kritisch hinterfragt werden, hätte sich selbst die Grundlagen entzogen "(Peglau, 2013, S. 517).

Peglau (2013) hat in seinem Buch „Unpolitische Wissenschaft? Wilhelm Reich und die Psychoanalyse im Nationalsozialismus" anhand vieler Dokumente und genauer Recherchen aufgezeigt, dass es während des Nationalsozialismus nicht zu einer gänzlichen Verdrängung der Psychoanalyse gekommen war.

„Wenn Psychoanalytiker zu Opfern des NS-Systems wurden, dann nie, weil sie Psychoanalytiker waren, sondern wegen ihrer jüdischen Herkunft oder widerständigen, insbesondere politisch ,linken "Äußerungen oder Aktivitäten" (Peglau, 2013, S. 502).

Reich war aktiver Analytiker und politischer Aktivist. Er war von 1924 bis 1930 Leiter des technisch-therapeutischen Seminars in Wien, ab 1925 Mitglied des Lehrinstituts der Vereinigung (vgl. Mühlleitner, 1992, S. 257), ein erfolgreicher psychoanalytischer Autor, in der Arbeiterfortbildung und in den Sexualberatungsstellen aktiv, Mitglied der sozialdemokratischen und später der kommunistischen Partei. Seine politische Tätigkeit wurde als zunehmend belastend für die Psychoanalyse $^{75}$ und die politischen Parteien, bei denen er Mitglied war, empfunden - das auch deshalb, weil er sich in seiner Kritik nicht vereinnahmen ließ, seine anfängliche Begeisterung für die politischen Entwicklungen in der

75 Peglau zitiert einen Brief von Freud an Eitingon vom 9.1.1932, in dem Freud „Reichs und Fenichels Versuch, die Zeitschriften für bolschewistische Propaganda zu missbrauchen“, kritisiert (Peglau, 2013, S. 133). Dabei ging es um den Artikel „Der masochistische Charakter. Eine sexualökonomische Widerlegung des Todestriebes und des Wiederholungszwanges“, der für die Veröffentlichung in der Internationalen Zeitschrift für Psychoanalyse vorgesehen war, Freuds Triebtheorie infrage stellte und, wie Peglau aus Freuds Tagebuch zitiert, zu einem langen Magenanfall Freuds führte (vgl. Peglau, 2013, S. 133). Reich bekam auch eine Absage für die Veröffentlichung seines Buches „Charakteranalyse“ im Internationalen Psychoanalytischen Verlag. Auszugsweise wird ein Brief vom 17.3. 1933 von Reich wiedergegeben, den Peglau zitiert: „Gestern teilte mir der Verlagsleiter, Herr Dr. [Martin] Freud, mit, dass auf Beschluss der Verlagskommission und der Verlagsinhaber der Vertrag, wonach mein Buch ,Charakteranalyse' im Verlag demnächst herauskommen sollte, rückgängig gemacht wird. Begründet wurde dieser Beschluss mit der Rücksicht auf die gegenwärtigen politischen Verhältnisse, die es nicht angebracht erschienen liessen [sic!], meinen kompromittierten Namen neuerdings offiziell zu vertreten“ (Peglau, 2013, S. 163). 
Sowjetunion etwa einer weniger enthusiastischen Sichtweise wich, die ihn in der dritten, korrigierten und erweiterten Auflage seines Buches zu einer veränderten Terminologie greifen ließ.

1930 verließ Reich Wien und ließ sich in Berlin nieder. Dort erreichte er einen großen Bekanntheitsgrad, wurde Mitglied der KPD, ,deren schlagkräftigen Agitations- und Propagandaapparat" er zur Verfügung hatte (Peglau, 2015, S. 170).

„Hinzu kam Reichs öffentlichkeitswirksames Engagement im Kampf gegen den Abtreibungsparagrafen 218, seine Dozententätigkeit an der weithin bekannten marxistischen Arbeiterschule und seine Leitungstätigkeit in einer KP-nahen, sexualreformerischen Massenorganisation - die er später als "Sexpol "bezeichnen sollte“" (Peglau, 2015, S. 170).

1942 schreibt Reich, auf jene Zeit zurückblickend:

„Schon 1929-1930 sperrte die österreichische Sozialdemokratie ihre Kulturorganisationen den Referenten unserer Organisation. Die sozialistischen sowohl wie die kommunistischen Organisationen verboten, trotz scharfen Protestes der Mitglieder, den Vertrieb der Schriften des ,Verlags für Sexualpolitik' in Berlin schon 1932. Mir wurde gedroht, daß ich an die Wand gestellt werden würde, sobald der Marxismus zur Macht in Deutschland gelangte. 1932 sperrten die kommunistischen Organisationen in Deutschland, gegen den Willen der Mitglieder, ihre Versammlungslokale für den sexualökonomischen Arzt" (Reich, 1986, S. 19).

Den Bücherverbrennungen fielen neben den Werken Freuds und seiner Tochter Anna nur die von Bernfeld und Reich zum Opfer; ein auf das gesamte Werk ausgedehntes Publikationsverbot traf Reich 1933 (vgl. Peglau, 2013, S. 503). Wie schon weiter oben ausgeführt, wurde das aufklärerische Wirken Reichs bei seinen Fortbildungsabenden, in denen es um Aufklärung der Jugendlichen, eine von Repressionen befreite Sexualität und die Emanzipation der Frauen ging, die im Sinne einer Selbstbemächtigung ihres Körpers und Lebens gegenläufig zu den autoritären Strukturen stand, von den Nationalsozialisten als besonders gefährlich erachtet.

Aus einem Brief von Reich an Anna Freud nach der Vorstandssitzung der WPV vom 21. 4. 1933 ist bei Peglau nachzulesen, dass Reich von sich aus nicht gewillt war, aus der Internationalen Psychoanalytischen Vereinigung auszutreten:

„Der Vorstand der Vereinigung forderte von mir mit Rücksicht auf die herrschende politische Situation die Einstellung meiner politischen Arbeit und soziologisch-wissenschaftlichen publizistischen Tätigkeit. [...] Ich erklärte, eine solche Zusage nicht machen zu können [...]“" (zitiert nach Peglau, 2013, S. 165). 
Reichs politische Tätigkeit führte 1933/34 auch zum Ausschluss aus der deutschen und Internationalen Psychoanalytischen Organisation, was Peglau zu folgendem Schluss führt: „Die Toleranz, die der Psychoanalyse und ihren Schriften im Nationalsozialismus gewährt wurde, beruhte also unter anderem darauf, dass die Psychoanalyseorganisation den Dissidenten Reich aus ihren Reihen entfernt hatte“ (Peglau, 2013, S. 505). Reich floh vor den Nationalsozialisten von Deutschland nach Dänemark, weiter nach Schweden und Norwegen und schließlich nach Amerika. Während der McCarthy-Ära wurde er inhaftiert, seine Bücher wurden im Jahre 1956 in den USA noch einmal verbrannt.

Peglau führt die fehlende Relevanz der Psychoanalyse in den letzten Jahrzehnten unter Verweis auf Richter oder Dahmer darauf zurück, dass mit dem Ausschluss Reichs 1933/34 aus der Internationalen Psychoanalytischen Vereinigung eine Entwicklung in Gang gesetzt wurde, durch die die gesellschaftskritische Funktion der Psychoanalyse aufgegeben wurde. ${ }^{76}$ Dazu gehören auch das fehlende Erinnern und Durcharbeiten der Rolle der Psychoanalyse in der NS-Zeit und nach dem Krieg und ihr Schweigen zu anderen relevanten politischen Ereignissen $^{77}$ (vgl. Peglau, 2013, S. 508 ff.).

„Die gegenwärtige Bedeutungsarmut der Psychoanalyse hat daher [...] mit dem durch die Analytiker selbst hergestellten und beibehaltenen, entpolitisierten 'Image der Analyse zu tun, mit der weitgehenden Verweigerung ihrer offiziellen Organisationen und der meisten ihrer Mitglieder, sich als Psychoanalytiker öffentlich an den gesellschaftskritischen Diskussionen zu beteiligen, sowie mit der Reduktion der Psychoanalyse auf Psychotherapie" (Peglau, 2013, S. 511).

So gesehen hat sich die Psychoanalyse - in Anspielung an das dem Abschnitt vorangestellte Zitat - eine ihrer wichtigen Grundlagen entzogen. Die Intention, die dieser Arbeit zugrunde liegt, ist unter anderem, an die Psychoanalyse als

76 Siehe dazu auch (Jacoby, 1985, S. 128 f.): „Der wachsende Druck des Faschismus war nun allenthalben zu spüren, bei der Gruppe um Fenichel insbesondere, nachdem Edith Jacobson 1936 verhaftet und in ein städtisches Gefängnis gesteckt worden war [...] Doch Freud hoffte immer noch, die österreichischen Behörden würden das Land und die Psychoanalyse dort vor dem Zugriff des Nationalsozialismus schützen. Um jedes Aufsehen zu vermeiden, bestimmte man 1935, dass Analytiker und Ausbildungskandidaten sich nicht an illegalen politischen Aktivitäten beteiligen sollten. Eine Kandidatin, Marie Langer, die später nach Argentinien emigrierte, geriet mit dieser Bestimmung in Konflikt und wurde um ein Haar von der Wiener Gruppe ausgeschlossen, nachdem die Polizei sie für kurze Zeit sistiert hatte.“

77, ,...] Reich wurde von der IPV nicht ausgeschlossen, weil er politisch aktiv war, sondern weil er eine ,gewisse“ politische Tätigkeit einforderte, die zu Konflikten mit dem NS-System geführt und so das Weiterbestehen der institutionalisierten Psychoanalyse in Deutschland zusätzlich gefährdet hätte“ (Peglau, 2013, S. 512). 
Methode der Kultur- und Gesellschaftskritik anzuknüpfen, um auf diese Weise mehr über das dem Individuum Unbewusste zu erfahren und es mit jenen Faktoren in Beziehung zu setzen, die es zu einem sozialen und politischen Wesen machen, das die Gesellschaft als Wirkfaktor an sich selbst erfassen kann.

\subsubsection{Otto Fenichel}

Nach Jacoby (1985) waren Fromm, Reich und Fenichel die ,produktivsten, leidenschaftlichsten und originellsten der politisch orientierten Freudianer" (1985, S. 48). Hier soll Fenichel nur mit einer seiner Arbeiten zu Wort kommen, nämlich mit jener über den Bereicherungstrieb (Fenichel, 1938). Darin behandelt er die Frage, in welchem Verhältnis die sozialen Institutionen zu den Trieben stehen, und erklärt diese Thematik als „entscheidend für die Bestimmung der Rolle der Psychologie beim Verstehen sozialer Prozesse“" (Fenichel, 1938, S. 213). Verkürzungen in Form von Biologisierungen, dass die sozialen Institutionen von den Trieben gleichsam hervorgebracht würden, lehnt er ab. Am Beispiel der Akkumulation von Geld bedeutet dies, dass erst bestimmte äußere Gegebenheiten, nämlich „Existenz und Funktion des Geldes im Gesellschaftssystem [...] diesen unspezifischen Triebregungen ihr spezifisches Objekt" geben (Fenichel, 1938, S. 211). Um welche Triebkomponenten handelt es sich dabei?

Zunächst um eine Form der Bedürfnisbefriedigung, deren Möglichkeiten in Abhängigkeit von soziökonomischen Gegebenheiten durchaus unterschiedlich ausfallen können. Interessant ist, wie innerhalb einer Gesellschaft, in der Reichtum unterschiedlich verteilt ist, die psychische Struktur diesen Gegebenheiten angepasst wird. Dazu gehören institutionelle Gewalt, illusionäre Befriedigungsvorstellungen und bestimmte Ideale wie „Sparsamkeit“ (vgl. Fenichel, 1938, S. 200).

„Die Kraft einer solchen Ideologie ist am stärksten bei jenen Klassen, deren Zukunftshoffnung sich aus der Erinnerung einer besseren Vergangenheit speist, besonders beim Kleinbürgertum, das durch den Fortschritt der Kapitalakkumulation ins Elend gestürzt wurde und nur durch Sparsamkeit das Verlorene wiederzugewinnen hofft. Anders ist das bei Proletariern, die nie irgend etwas ihr eigen nannten [...] Die gerühmte ,proletarische Solidarität', die Bereitschaft, auch die geringste Habe zu teilen, erscheint uns nicht so sehr als eine rühmenswerte Tugend, sondern als ein rationaler Ausdruck des Sachverhalts [...] Die Versuche zu sparen lassen sich tatsächlich nicht realisieren " (Fenichel, 1938, S. 200 f.).

Bei der zweiten Triebquelle handelt es sich um das Streben nach Macht und Status, um narzisstische Strebungen, die der Regulation des Selbstwertgefühls dienen. 
„,Das ursprüngliche Triebziel sind nicht die Reichtümer, sondern ist der Wunsch, Macht und Achtung anderer Menschen und seiner selbst zu genießen. Erst eine Gesellschaft, in der Macht und Achtung auf Geldbesitz beruhen, macht aus diesem Bedürfnis nach Macht und Achtung ein Bedürfnis nach Reichtum “ (Fenichel, 1938, S. 204).

Die dritte Triebquelle wird als Besitzstreben identifiziert, zurückgeführt auf die analen Bestrebungen des Zurückhaltens und Ausstoßens. Im Gegensatz zu Ferenczi ist für Fenichel das Besitzstreben, das dem Kapitalismus immanent ist, nicht allein libidinös motiviert, sondern

„,ein solches Gesellschaftssystem bedient sich jener Triebregungen, die der Notwendigkeit zu akkumulieren nützlich sind, und stärkt sie "78 (Fenichel, 1938, S. 207). Dabei werden die gesellschaftlichen Faktoren meistens verdeckt (vgl. Fenichel, 1938, S. 207).

Die ,gesellschaftliche Matrix“ (Fenichel, 1938, S. 210) schließlich ist die vierte Komponente, die Fenichel im Zusammenhang mit dem Bereicherungstrieb anführt. Es ist dies das kapitalistische Wirtschaftssystem, das mit der Akkumulation von Kapital dem Selbsterhaltungstrieb entgegenkommt und als Ideologie der herrschenden Klasse auch von den anderen Klassen angestrebt wird. „Die Konkurrenz zwingt den Kapitalisten bei Strafe seines eigenen Untergangs, die Produktion auf das äußerste zu steigern“ (Fenichel, 1938, S. 199). Der Psychoanalytiker begibt sich hier auf das Gebiet der Soziologie, wenn er der Frage nachgeht, welche Beziehungen zwischen ökonomischem System und Trieben bestehen (vgl. Fenichel, 1938, S. 200 ff.). Dabei erscheint Fenichel die marxistische Zugangsweise mit den Thesen, dass die Ideologie einer Gesellschaft durch die ökonomischen Bedingungen gestaltet wird und als solche über die Handlungen der Menschen wieder auf die ökonomische Basis zurückgreife, zu allgemein. „Sie [die Thesen] lassen sich spezifizieren, wenn wir den Mechanismus dieser Wechselwirkungen verständlich machen können, und dabei kann uns nur die Psychoanalyse helfen“" (Fenichel, 1938, S. 216).

So wenig wie der Kapitalismus „Resultat einer ,analerotischen Mutation“ war, die vom Himmel fiel““ (1938, S. 217), ist für Fenichel klar: Die

„Erfindung des Geldes und Veränderungen in der Funktion des Geldes waren nur möglich bei einer bestimmten Intensität und vor allem bei einem bestimmten Grad der Unterdrückung analerotischer Triebe. Aber diese Triebabwehr muß ihrerseits eine Vorgeschichte und materielle Determinanten haben " (Fenichel, 1938, S. 217).

78 Hervorhebung durch Fenichel. 
Damit wird der Bereicherungstrieb in seiner historischen Dimension erfasst und aus der anthropologischen Fixierung gelöst.

\subsubsection{Erich Fromm}

Fromm sieht in der Tatsache, dass der Mensch, vereinfacht gesprochen, Natur ist, das heißt von biologischen Faktoren bestimmt wird, aber nicht über die Instinkthaftigkeit der Tiere verfügt, die spezifischen Bedingungen der menschlichen Existenz. Gleichzeitig ist der Mensch als einziges Wesen sich seiner selbst bewusst und kann über seine eigene Existenz reflektieren. In einer ,sozio-biologischen Ausrichtung" (Fromm, 1990c, S. 19) untersucht Fromm daher neben den konstitutionellen biologischen Einflussfaktoren auch jene, die aus dem historischen Kontext entstehen.

„Ich [...] entwickelte eine Theorie, in deren Mittelpunkt die Bedürfnisse stehen, die sich aus den Existenzbedingungen des Menschen ergeben. Ich betone die Rolle der Gesellschaft, und zwar nicht als ,Kultur', sondern als eine bestimmte Gesellschaft, die entsprechend den Maximen ihrer Produktionsweise und ihrer wichtigsten Produktivkräfte strukturiert ist. Darüber hinaus betone ich die Bedeutung von Werten und ethischen Fragen für das Verstehen des Menschen "(Fromm, 1990a, S. 35).

$\mathrm{Zu}$ den besonderen psychischen Bedürfnissen des Menschen, die aus den existenziellen Bedingungen erwachsen, gehören das Bezogensein auf andere, das Bedürfnis, verwurzelt zu sein, geboren zu werden und gleichzeitig in den Mutterleib zurückkehren zu wollen, das Bedürfnis nach Transzendenz, nach Identität und das Bedürfnis nach einem Rahmen der Orientierung und einem Objekt der Hingabe (vgl. Fromm, 1956, S. 99 ff.).

Fromm sieht sich mit seiner Theorie nicht in Gegnerschaft zur Psychoanalyse Freuds, sondern versteht sie als Weiterentwicklung derselben, auch indem er die Psychoanalyse selbst auf die Theorienbildung anwendet (vgl. Fromm, 1990a, S. 40). Das bedeutet, dass dem Verdrängten innerhalb der Theorienbildung besonderes Augenmerk geschenkt wird. Trotz seiner Kritik am „mechanistischphysiologischen" Grundverständnis der Libidotheorie lehnt Fromm eine Zuordnung seiner Arbeiten zu den sogenannten kulturellen Ausrichtungen oder „Kulturalisten“, „Neo-Freudianern“ oder „Revisionisten“, zu denen Harry Stuck Sullivan oder Karen Horney gerechnet werden, ab (vgl. Fromm, 1990a, S. 34 f.). ${ }^{79}$

79 Rainer Funk (1992) zitiert im Vorwort zu „Gesellschaft und Seele“ einen Brief, den Erich Fromm am 18.12.1936 an Karl August Wittfogel geschrieben hat: ,[...] ich versuche zu zeigen, daß die Triebe, die gesellschaftliche Handlungen motivieren, nicht, wie Freud annimmt, Sublimierungen der sexuellen Instinkte sind, sondern Produkte des gesellschaftlichen Prozesses, oder genauer gesagt, Reaktionen auf be- 
In seiner „dialektischen Revision der Psychoanalyse“ (Fromm, 1990a, S. 40) sucht Fromm nach den Ursachen der krankmachenden Gefühle wie „Entfremdung, Angst, Einsamkeit, [...] Angst vor tiefgreifenden Gefühlen, [...] Mangel an Tätigsein und [...] das Fehlen von Freude" (Fromm, 1990a, S. 37) in den gesellschaftlichen Bedingungen. Diese Gefühle haben seiner Meinung nach die Symptome, die durch die Verdrängung der Sexualität zu Freuds Zeiten hervorgerufen wurden, abgelöst.

Fromm zeigt sich kritisch gegenüber der Gleichsetzung des Unbewussten mit den verdrängten triebhaften Aspekten des Menschen, vorwiegend mit dem Sexualtrieb, da dadurch andere verdrängte Inhalte wie das gesellschaftliche Unbewusste, das weiter oben erläutert wurde, oder die Verdrängung der Mutterbindung aus dem Blickfeld geraten. Letztere bringt er in Verbindung mit dem Wunsch, lebenslang in Abhängigkeit zu verbleiben, beschützt zu werden, was auch in der Verschiebung der Mutterrepräsentanz auf Institutionen zum Ausdruck kommen kann (vgl. Fromm, 1990a, S. 58 f.). In extremerer Form kann sich diese Mutterbindung im Wunsch ausdrücken, in den Mutterleib zurückkehren zu wollen, gar nicht geboren worden zu sein. „Dann wird der Mutterleib zum Grab, zur Mutter Erde, in der man ,begraben' werden, zum Meer, in dem man ertrinken möchte" (Fromm, 1990a, S. 59). Für Fromm sind diese Wünsche nicht Ausdruck von ödipalen Strebungen, „die inzesthaften Strebungen stellen oft den Versuch dar, sich vor der tieferen und lebensbedrohlichen Sehnsucht nach der Mutter zu schützen“ (Fromm, 1990a, S. 59). Mit diesen Gedanken nimmt Fromm Weiterentwicklungen im Bereich der Psychoanalyse vorweg, die, wie die Bindungstheorie, die Bedeutung dieser frühen Beziehung für beiderlei Geschlechter herausgearbeitet haben.

Für Fromm sind sowohl die Sehnsucht nach der Mutter als auch die Sehnsucht nach „Idolen“, nach „magischen Helfern“ (Fromm, 1990a, S. 74), denen man sich anvertrauen kann und die Stärke vermitteln, Ausdruck der oben beschriebenen existenziellen Widersprüchlichkeit des Menschen.

„Solche ,magischen Helfer ' können alle Arten von religiösen Idolen sein oder auch Naturgewalten oder Institutionen und Gruppen (wie der Staat oder die Nation); sie können charismatische oder auch nur mächtige Führer sein; sie können einfache

stimmte Konstellationen, unter denen der Mensch seine Instinkte befriedigen muß. Diese Triebe, die ich in solche teile, die sich auf die Beziehungen zum Menschen (Liebe, Haß, Sadomasochismus), und solche, die sich auf die Art der Aneignung (Trieb zum Empfangen, Wegnehmen, Sparen, Sammeln und Produzieren) beziehen, sind grundsätzlich verschieden von den naturalen Faktoren, nämlich den Instinkten Hunger, Durst, Sexualität. Während diese allen Menschen und Tieren gemeinsam sind, sind jene spezifisch menschliche Produkte und nicht biologisch, sondern aus der gesellschaftlichen Lebenspraxis heraus zu verstehen.“ 
Menschen wie der Vater und die Mutter, der Ehemann oder die Ehefrau usw. sein " (Fromm, 1990a, S. 65).

In Abgrenzung zu Freud sieht Fromm die Ursachen für die Mächtigkeit der Idole nicht in einer Wiederholung der frühen Bindung an die Elternfiguren, also in einer Übertragungsbeziehung, sondern er sieht sie als Ausdruck der „,conditio humana“, das heißt der Tatsache, dass ,die meisten Menschen sich tief in ihrem Unbewußten wie Kinder fühlen und sich deshalb nach einer mächtigen Figur sehnen, der sie vertrauen und der sie sich unterwerfen können" (Fromm, 1990a, S. 75). Sowohl die psychische Struktur eines Menschen als auch äußere Lebensbedingungen nähren die Bindung an Idole, die in Krisenzeiten offensichtlich wird.

„Im gesellschaftlichen Bereich sind solche traumatischen Ereignisse zum Beispiel schwere ökonomische Krisen, die zu Massenarbeitslosigkeit führen, eine galoppierende Inflation, starke Ungewißheitszustände (wie etwa die Krise von 1929, die in Deutschland zum Emporkommen von Hitler geführt hat) oder Krieg " (Fromm, 1990a, S. 68).

Die Struktur einer Gesellschaft kann die Idolbildung verstärken oder auch mindern - Letzteres, wenn mehr Transparenz, individuelle Freiheit und soziale Gerechtigkeit herrschen.

Aufgabe einer ,analytischen Sozialpsychologie“ ist es nach Fromm, zu zeigen,

„, daß die Produktions- und Lebensweise der Menschen eine ganz bestimmte Charakterstruktur schafft, und daß das Bewußtsein der Menschen, soweit es nicht unmittelbar ein rationaler Reflex der gesellschaftlichen Praxis ist, durch die besondere Gestalt der Triebe, Ängste und Erwartungen des Menschen - speziell der unbewußtenbedingt ist" (Fromm, 1937, S. 93).

Dieser Aufgabe gilt es in Bezug auf die spezifischen sozioökonomischen Verhältnisse, innerhalb derer sich die Finanzkrise 2008 abspielte, hypothesenbildend nachzukommen. 
Open Access Dieses Kapitel wird unter der Creative Commons Namensnennung 4.0 International Lizenz (http://creativecommons.org/licenses/by/4.0/deed.de) veröffentlicht, welche die Nutzung, Vervielfältigung, Bearbeitung, Verbreitung und Wiedergabe in jeglichem Medium und Format erlaubt, sofern Sie den/die ursprünglichen Autor(en) und die Quelle ordnungsgemäß nennen, einen Link zur Creative Commons Lizenz beifügen und angeben, ob Änderungen vorgenommen wurden.

Die in diesem Kapitel enthaltenen Bilder und sonstiges Drittmaterial unterliegen ebenfalls der genannten Creative Commons Lizenz, sofern sich aus der Abbildungslegende nichts anderes ergibt. Sofern das betreffende Material nicht unter der genannten Creative Commons Lizenz steht und die betreffende Handlung nicht nach gesetzlichen Vorschriften erlaubt ist, ist für die oben aufgeführten Weiterverwendungen des Materials die Einwilligung des jeweiligen Rechteinhabers einzuholen.

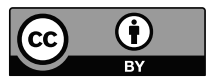

